\title{
Effects of Navier Slip on Entropy Generation in a Porous Channel with Suction/Injection*
}

\author{
Adetayo Samuel EEGUNJOBI** and Oluwole Daniel MAKINDE** \\ ${ }^{* *}$ Institute for Advance Research in Mathematical Modelling and Computation, Cape Peninsula, \\ University of Technology, P.O.Box 1906, Bellville 7535, South Africa. \\ E-mail: samdet1@yahoo.com \\ E-mail:makinded@cput.ac.za
}

\begin{abstract}
In this paper, we investigate the combined effects of suction/injection and asymmetric Navier slips on the entropy generation rate in a steady flow of an incompressible viscous fluid through a porous channel subjected to non-uniform temperature at the walls. The nonlinear model problem is tackled numerically using shooting quadrature. Both the velocity and temperature profiles are obtained and utilized to compute the entropy generation number. The effects of slip parameter, Brinkmann number, the Peclet number and suction/injection Reynolds number on the fluid velocity, temperature profile, skin friction, Nusselt number, entropy generation rate and Bejan number are depicted graphically and discussed quantitatively.
\end{abstract}

Key words: Porous Channel, Asymmetric Navier Slip, Heat Transfer, Entropy Generation, Bejan Number

\section{Introduction}

Rapid progress in science and technology has led to the development of an increasing number of flow devices that involve the manipulation of fluid flow in various geometries. Many textbooks of fluid dynamics fails to mention that the no-slip condition remains an assumption due to unusual agreement with experimental results for a century. Nevertheless, another approach was supposed that fluid can slide over a solid surface because the experimental fact was not always accepted in the past. Navier (1823) proposed general boundary conditions which include possibility of fluid slip at the solid boundary. He proposed that velocity at a solid surface is proportional to the shear stress at the surface. The phenomenon of slip occurrence has been demonstrated by the recent theoretical and experimental studies, these include; Sahraoui and Kaviany (1994), Buckingham et al. (2003), Berh (2004), Raoufpanah (2005), Chauhan and Kumar (2009), Tripathi et al. (2011), Gupta (2011), etc.

Moreover, entropy generation in engineering and industrial flow systems provides insight into the power consumption through thermodynamic losses. Therefore, the entropy minimization provides power optimization for the fluid motion in the porous channel. Efficient energy utilization during the convection in any fluid flow is one of the fundamental problems of the engineering processes to improve the system. Consequently, investigation of entropy generation due to combined effects of wall suction/injection, Navier slip and non-uniform surface temperature become essential. In a pioneering work, Bejan (1982) presented the analysis of thermodynamic second-law to inherent irreversibility 
in heat transfer with respect to thermal design of an engineering system. Thereafter, considerable research studies were carried out to investigate entropy generation such as: Bejan (1996), Sahin (2000), Sahin and Ben-Mansom(2003), Makinde and Aziz(2010), Makinde (2010), Ozalp (2010), Erbay et al(2003), Vazquez et al. (2011), Haddad et al (2004), e.t.c.

Meanwhile, fluid flow through a porous channel has been studied theoretically and experimentally by numerous authors due to its wide applications in various fields such as diffusion technology, transpiration cooling, hemodialysis processes, desalination, flow control in nuclear reactors, etc. In a pioneering work, Berman (1953) presented an exact solution of the Navier-Stokes equations that describes the steady two-dimensional flow of an incompressible viscous fluid along a channel with parallel rigid porous walls, the flow being driven by uniform suction or injection at the walls. Thereafter, many authors such as Terrill (1964, 1965), Makinde (1998), Robinson (1976), Brady (1984), Makinde (2011), etc., have extended and reconsidered the problem under various physical conditions. To the best of authors' knowledge, none of these papers have addressed combined effects of wall suction/injection and asymmetric Navier slips on steady flow in a porous channel with non uniform walls temperature.

In the present study, our objective is to investigate the combined effects of wall suction/injection and asymmetric Navier slips on an incompressible viscous flow in a porous channel subjected to non-uniform walls temperature. The work essentially extends the recent work of Ajibade et al. (2011) to include asymmetric Navier slip and non-uniform wall temperature. The nonlinear model problem is tackled numerically. The paper is organized as follows: in section 2, we define the problem, given the governing equations and present the mathematical formulation. In section 3, we derive the entropy generation rate, skin friction, Nusselt number and Bejan number. In section 4, we present and discuss the pertinent results graphically and quantitatively. Finally, a concluding remark is given in section 5 .

\section{Mathematical Model}

We consider steady flow of an incompressible viscous fluid through a uniformly porous channel under the combined action of constant axial pressure gradient, wall suction / injection and asymmetric Navier slip as shown in figure 1 below,

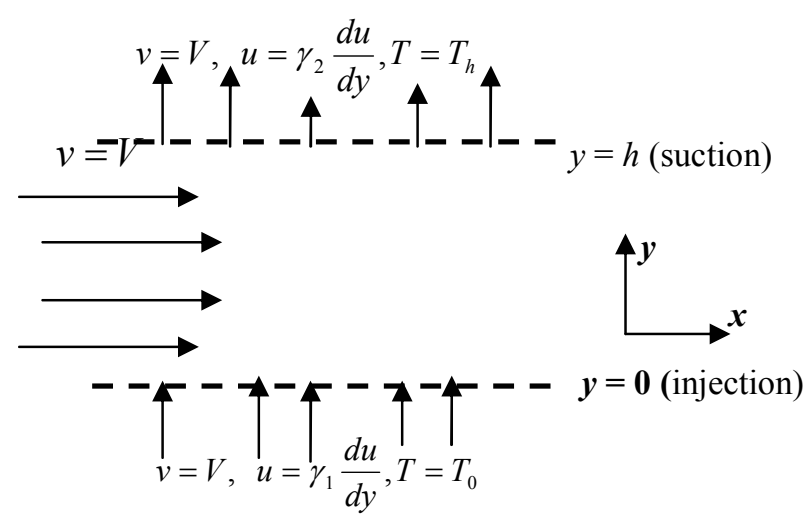

Figure 1: Schematic diagram of the problem

The fluid equations for momentum and energy balance governing the problem under consideration can be written as Ajibade et al. (2011): 


$$
V \frac{d u}{d y}=-\frac{1}{\rho} \frac{d P}{d x}+\frac{\mu}{\rho} \frac{d^{2} u}{d y^{2}}
$$

and

$$
V \frac{d T}{d y}=\alpha \frac{d^{2} T}{d y^{2}}+\frac{\mu}{\rho c_{P}}\left(\frac{d u}{d y}\right)^{2} .
$$

The boundary conditions are

$$
\begin{aligned}
& u(0)=\gamma_{1} \frac{d u(0)}{d y}, T(0)=T_{0}, \\
& u(h)=\gamma_{2} \frac{d u(0)}{d y}, T(h)=T_{h},
\end{aligned}
$$

where (1) and (2) are momentum and energy equations respectively, $h$ is the channel width, $u$ is the velocity of the fluid, $P$ is the fluid pressure, $V$ is the uniform suction / injection velocity at the channel walls, $\mu$ is the fluid viscosity, $\alpha$ is the thermal diffusivity, $k$ is the thermal conductivity coefficient, $c_{P}$ is the specific heat at constant pressure, $T$ is the temperature, $\gamma_{1}$ and $\gamma_{2}$ are slip coefficients. We introduce the following non-dimensional quantities:

$$
\theta=\frac{T-T_{0}}{T_{h}-T_{0}}, \alpha=\frac{k}{\rho c_{p}}, w=\frac{u}{V}, \bar{P}=\frac{h P}{\mu V}, \eta=\frac{y}{h}, X=\frac{x}{h}, G=-\frac{d \bar{P}}{d X} .
$$

Substituting equation (4) into equations (1)-(3), we obtain

$$
\begin{aligned}
& \frac{d^{2} w}{d \eta^{2}}-\operatorname{Re} \frac{d w}{d \eta}+G=0, \\
& \frac{d^{2} \theta}{d \eta^{2}}-\mathrm{P} e \frac{d \theta}{d \eta}+B r\left(\frac{d w}{d \eta}\right)^{2}=0,
\end{aligned}
$$

with the boundary conditions

$$
\left.\begin{array}{ll}
w(0)=\beta_{1} \frac{d w(0)}{d \eta}, & w(1)=\beta_{2} \frac{d w(1)}{d \eta} \\
\theta(0)=0, & \theta(1)=1
\end{array}\right\}
$$

where $G$ is the pressure gradient parameter,

$\operatorname{Re}=\frac{V h \rho}{\mu}($ Reynolds number $)$,

$P e=\operatorname{Pr} \operatorname{Re}=\frac{V h}{\alpha}($ Peclet number $)$

$\operatorname{Pr}=\frac{\mu}{\alpha \rho}($ Prandtl number $)$ 
$B r=\frac{\mu V^{2}}{\rho c_{p} \alpha\left(T_{h}-T_{0}\right)}$ (Brinkmann number $)$,

$\beta_{1}=\frac{\gamma_{1}}{h}$ (lower plate slip parameter),

$\beta_{2}=\frac{\gamma_{2}}{h}$ (upper plate slip parameter).

Moreover, equation (5) subject to boundary conditions in (7) can be solved exactly and we obtain

$$
\begin{aligned}
& w(\eta)=\frac{e^{\mathrm{Re} \eta} G\left(1-\beta_{2}+\beta_{1}\right)}{\operatorname{Re}\left(1-e^{\mathrm{Re}}-\beta_{1} \operatorname{Re}+e^{\operatorname{Re}} \beta_{2} \operatorname{Re}\right)}+\frac{\eta G}{\operatorname{Re}} \\
& +\frac{G\left(\beta_{1} \operatorname{Re}+\operatorname{Re} \beta_{1} \beta_{2} e^{\operatorname{Re}}-\beta_{1} \beta_{2} \operatorname{Re}-e^{\operatorname{Re}} \beta_{1}+\beta_{2}-1\right)}{\operatorname{Re}\left(1-e^{\operatorname{Re}}-\beta_{1} \operatorname{Re}+e^{\operatorname{Re}} \beta_{2} \operatorname{Re}\right)} .
\end{aligned}
$$

The above coupled nonlinear boundary value problem represented by equations (5)-(6) together with their boundary conditions in equation (7) have been solved numerically using the shooting iteration technique together with Runge-kutta fourth-order integration scheme, Nachtshein and Swigert (1965).

\section{Entropy Generation}

Fluid flow and heat transfer processes inside a porous narrow channel are irreversible. The non-equilibrium conditions arise due to the exchange of energy and momentum within the fluid and at porous solid boundaries, thus resulting in entropy generation. Following Bejan (1996), the volumetric entropy generation rate is given as

$$
E_{G}=\frac{k}{T_{0}^{2}}\left(\frac{d T}{d y}\right)^{2}+\frac{\mu}{T_{0}}\left(\frac{d u}{d y}\right)^{2},
$$

where the first term on the right side of equation (9) is the irreversibility due to heat transfer and the second term is the entropy generation due to viscous dissipation. Using equation (4), we express equation (9) in dimensionless form as

$$
N_{S}=\frac{T_{0}^{2} h^{2} E_{G}}{k\left(T_{h}-T_{0}\right)^{2}}=\left(\frac{d \theta(\eta)}{d \eta}\right)^{2}+\frac{B r}{\Omega}\left(\frac{d \eta(\eta)}{d \eta}\right)^{2},
$$

where $\Omega=\left(T_{h}-T_{0}\right) / T_{0}$ is the temperature difference parameter and

$$
N_{1}=\left(\frac{d \theta(\eta)}{d \eta}\right)^{2}, N_{2}=\frac{B r}{\Omega}\left(\frac{d w(\eta)}{d \eta}\right)^{2}
$$

The Bejan number is given as

$$
B e=\frac{N_{1}}{N_{s}} \text {. }
$$

It is noteworthy that the Bejan number ranges from 0 to 1 and $B e=0$ is the limit where the irreversibility is dominated by fluid friction effects. $B e=1$ is the limit where the irreversibility due to heat transfer dominates the flow system by virtue of finite temperature differences. The contribution of both heat transfer and fluid friction to entropy generation 
are equal when $B e=1 / 2$.

\section{Results and Discussion}

In order to validate our results, we have chosen physically meaningful values for the parameters. The Prandtl number was taken in the range of $\operatorname{Pr}=0.71$ to 7.1 which corresponds to Prandtl number in the range of air and that of water. From our model in Fig. 1, it is important to note that the fluid suction takes place at the upper wall while the fluid injection occurs at the lower wall simultaneously. In order to validate the accuracy of our numerical procedure, the exact solution obtained in equation (8) is compared with the numerical solution obtained using shooting iteration technique together with Runge-kutta fourth-order integration scheme as illustrated in table 1. A perfect agreement is achieved between the numerical and exact solutions.

Table 1: Computation showing comparison between the exact and numerical solution of velocity profile for $\mathrm{G}=1, \mathrm{Re}=\beta_{1}=\beta_{2}=0.1$

\begin{tabular}{|c|c|c|}
\hline$\eta$ & $\begin{array}{c}\text { Exact Solution } \\
w(\eta)\end{array}$ & $\begin{array}{c}\text { Numerical } \\
\text { Solution } w(\eta)\end{array}$ \\
\hline 0 & 0.03956249 & 0.03956249 \\
\hline 0.1 & 0.07430662 & 0.07430662 \\
\hline 0.2 & 0.09934983 & 0.09934983 \\
\hline 0.3 & 0.11459457 & 0.11459457 \\
\hline 0.4 & 0.11994234 & 0.11994234 \\
\hline 0.5 & 0.11529370 & 0.11529370 \\
\hline 0.6 & 0.10054817 & 0.10054817 \\
\hline 0.7 & 0.07560428 & 0.07560428 \\
\hline 0.8 & 0.04035953 & 0.04035953 \\
\hline 0.9 & -0.00528959 & -0.005289591 \\
\hline 1.0 & -0.06144767 & -0.061447671 \\
\hline
\end{tabular}

Fig 2 depicts the effects of increase in Reynolds number (Re). As Re increases, fluid injection into the channel through the lower wall of the channel increases while the rate of fluid suction at the upper wall of the channel increases as well. This leads to a decrease in the fluid velocity at the lower channel wall region and an increase in flow reversal (represented by negative velocity) at the upper wall region $(\eta=1)$.

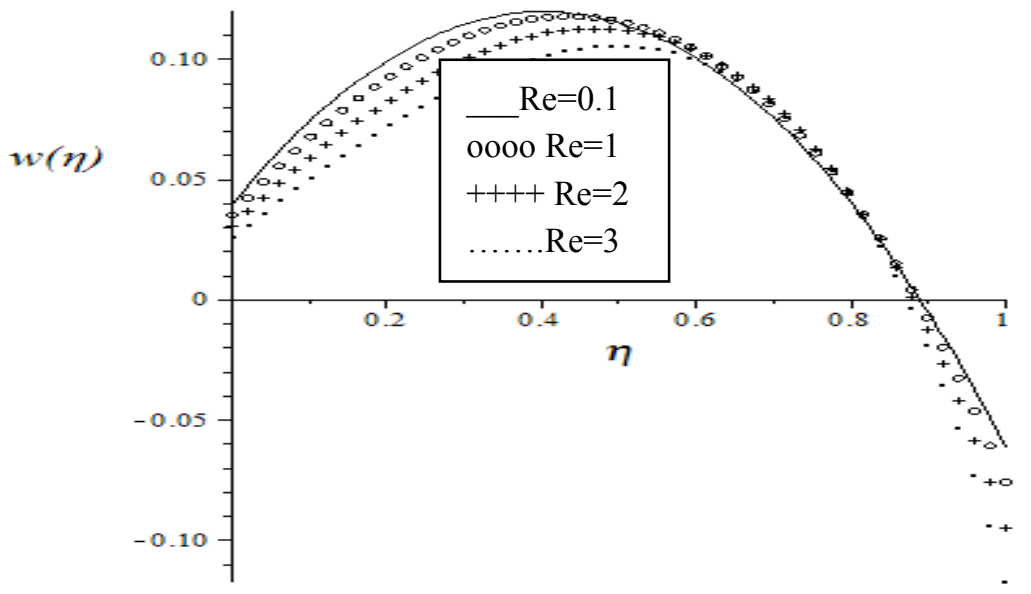

Figure 2: Velocity profile, $\beta_{1}=\beta_{2}=0.1, \mathrm{G}=1, \mathrm{Pr}=0.71, \mathrm{Br}=0.1$ 
Fig 3 shows velocity profile while $\beta_{1}$ is increasing. An increase in slip coefficient $\beta_{1}$ at the lower wall causes an increase in the velocity at the injection wall while the velocity reversal at suction wall increases slightly. In Fig 4 we observed that an increase in the slip coefficient $\beta_{2}$ at the upper wall causes a little decrease in velocity at injection wall while a large decrease in the fluid velocity is noticed with a high rate of flow reversal at the suction wall.

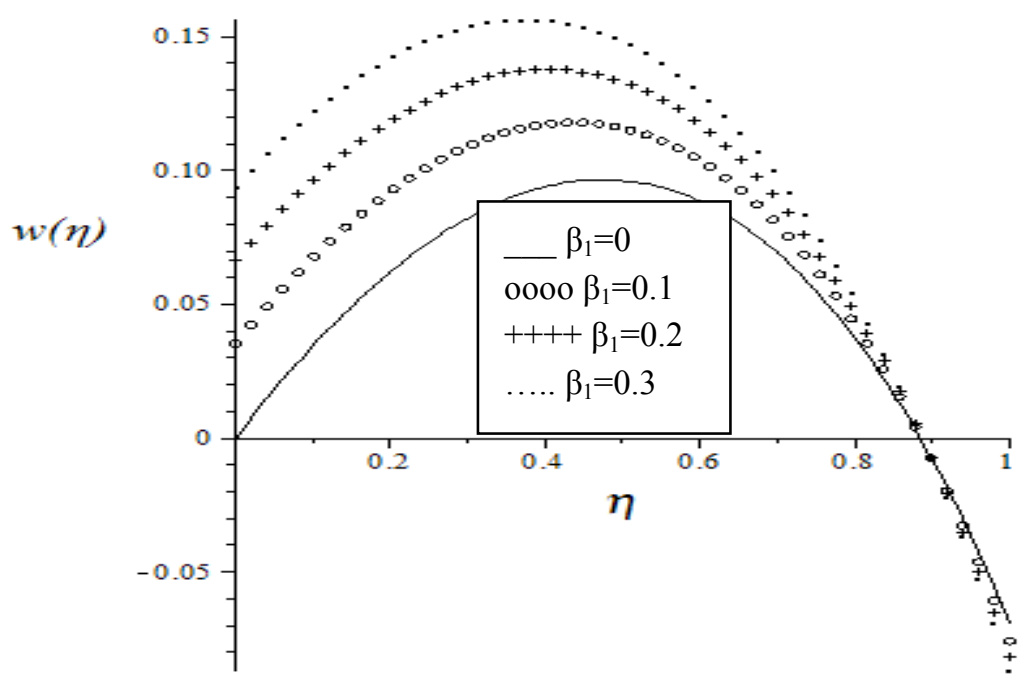

Figure 3: Velocity profile, $\beta_{2}=0.1, \mathrm{G}=\mathrm{Re}=1, \mathrm{Pr}=0.71, \mathrm{Br}=0.1$

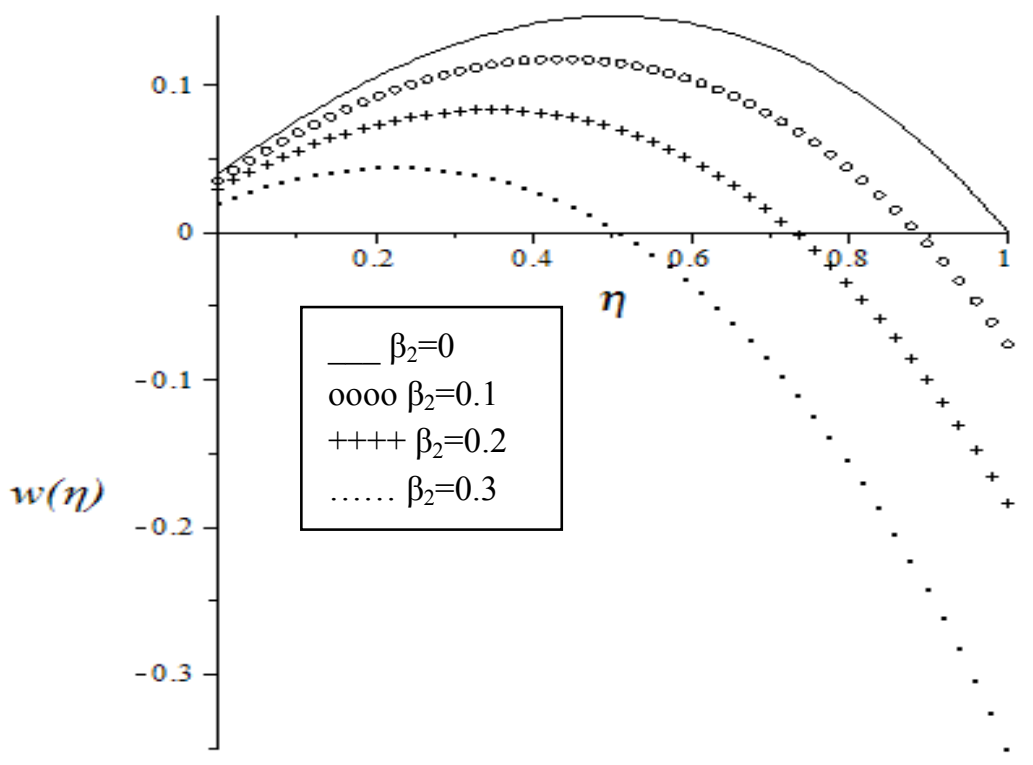

Figure 4: Velocity profile, $\beta_{1}=0.1, \mathrm{G}=\mathrm{Re}=1, \operatorname{Pr}=0.71$

Fig 5 depicts the variation in velocity profile with increasing values of pressure gradient parameter. As the pressure gradient increases, the velocity at injection wall increases and attains its maximum value along the channel centerline region. A slight increase in the reverse flow appears at suction wall. 


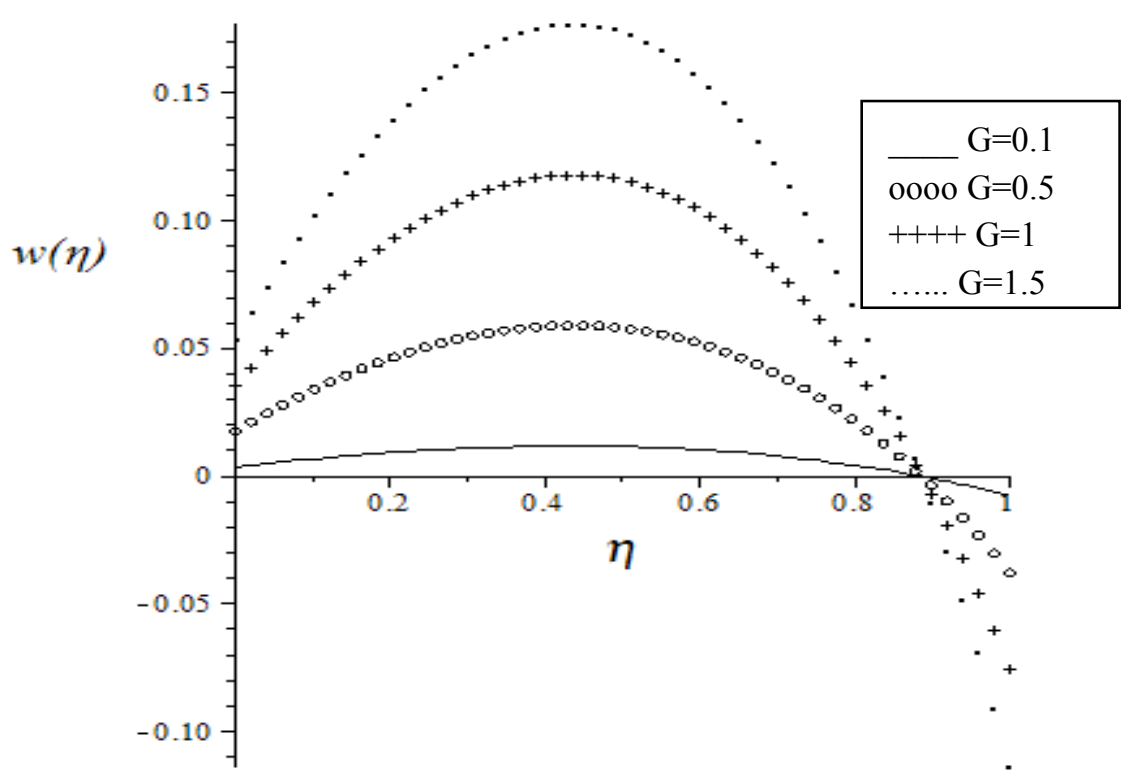

Figure 5: Velocity profile, $\beta_{1}=\beta_{2}=0.1, \operatorname{Re}=1, \operatorname{Pr}=0.71, \mathrm{Br}=0.1$

Fig. 6 shows temperature profile when there is increment in Brinkmann (Br) number due to viscous dissipation effects. The fluid temperature increases with an increase $\mathrm{Br}$ with minimum value at the injection wall and maximum value at suction wall satisfying the boundary condition. Fig.7 depicts temperature profile when the Prandtl number is increasing from 0.71 (air) to 7.1 (water). Increase in Prandtl number causes a sporadic decrease in temperature at the injection wall region and a slight decrease in temperature at the suction wall region. Similar trend is observed in fig. 8 as the Reynolds number increases. A slight decrease in the fluid temperature is noticed with increasing value of Reynolds number.

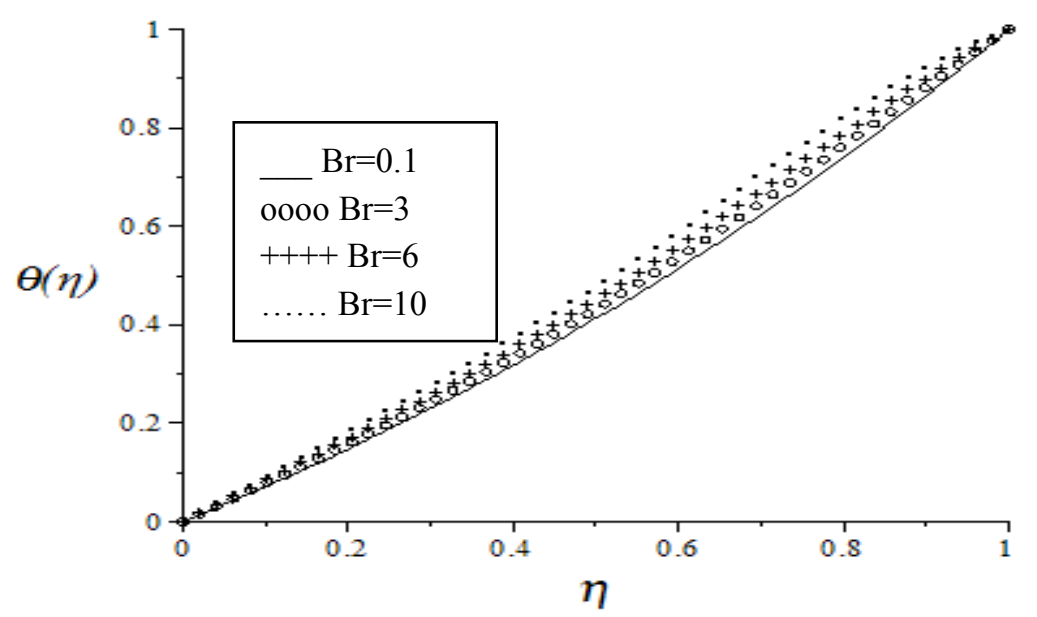

Figure 6 Temperature profile, $\beta_{1}=\beta_{2}=0.1, \mathrm{G}=\mathrm{Re}=1, \operatorname{Pr}=0.71$ 


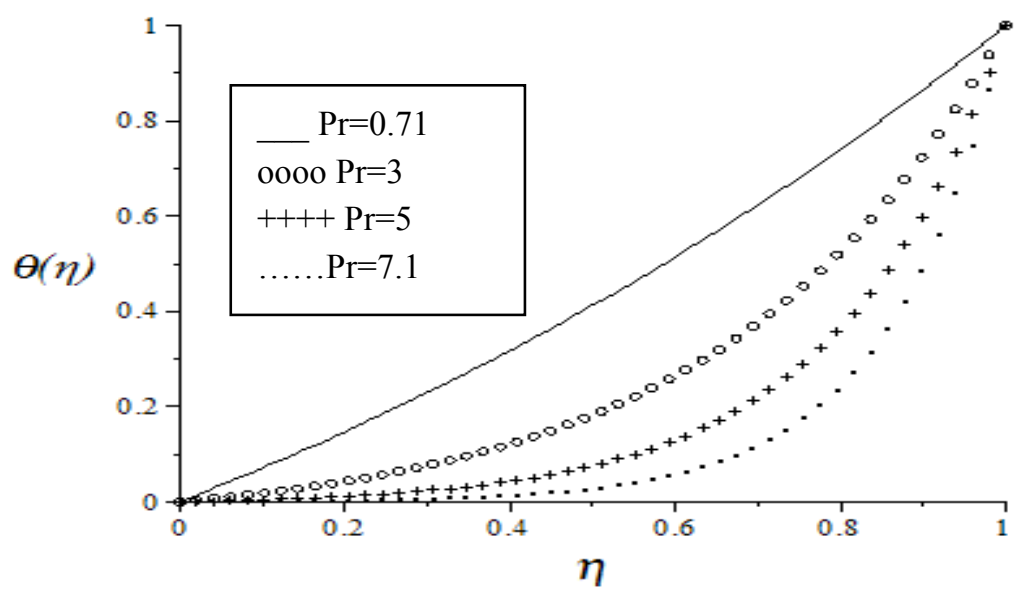

Figure 7: Temperature profile, $\beta_{1}=\beta_{2}=\mathrm{Br}=0.1, \mathrm{G}=\mathrm{Re}=1$

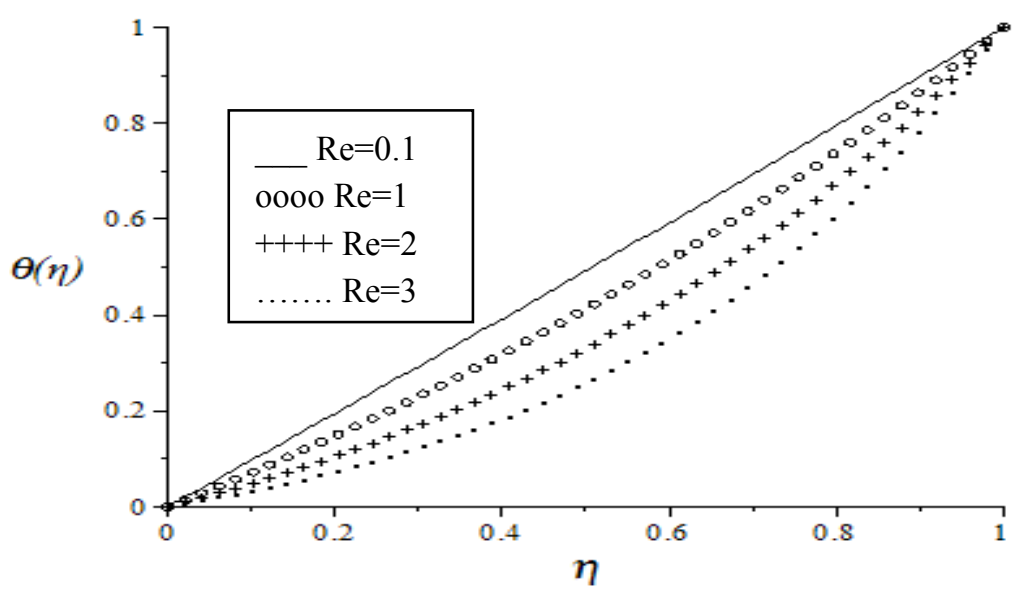

Figure 8 Temperature profile, $\beta_{1}=\beta_{2}=0.1, \mathrm{G}=1, \operatorname{Pr}=0.71, \mathrm{Br}=0.1$

Fig.9 illustrates the entropy generation rate when Reynolds number is increasing and other parameters remain constant. The entropy production at the injection walls decreases, however, at suction wall, entropy generation rate increases. Fig.10 depicts entropy generation rate when group parameter $\mathrm{Br} \Omega^{-1}$ increases due to a combined decrease in the temperature difference parameter and an increase in the viscous heating. As $\mathrm{Br} \Omega^{-1}$ increases, the entropy generation rate at both walls increases but more at suction wall. The entropy generation is lowest within the channel centerline region. In fig.11, an increase in Prandtl number decreases the rate of entropy production at the injection wall but increase entropy generation rate at the suction wall. Figs.12-13 depict entropy generation rate when the slip coefficients are increasing while other parameters remain constant. Increase in $\beta_{1}$ and $\beta_{2}$ decrease entropy generation rate at the injection wall and increase entropy generation rate at the suction wall. Fig.14 shows the effect of an increase in pressure gradient parameter $(G)$ on entropy generation rate. As $\mathrm{G}$ increases, an increase in entropy production is observed at both walls but the increment in entropy generation is more at suction wall. 


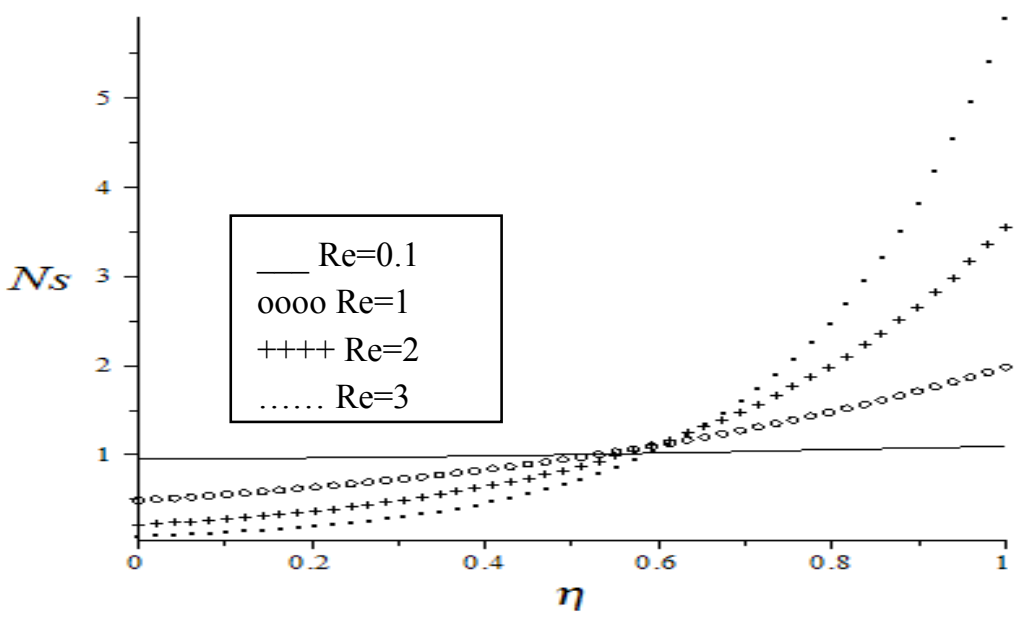

Figure 9: Entropy generation rate, $\mathrm{Br} \Omega^{-1}=\beta_{1}=\beta_{2}=\mathrm{Br}=0.1, \mathrm{G}=1, \mathrm{Pr}=0.71$

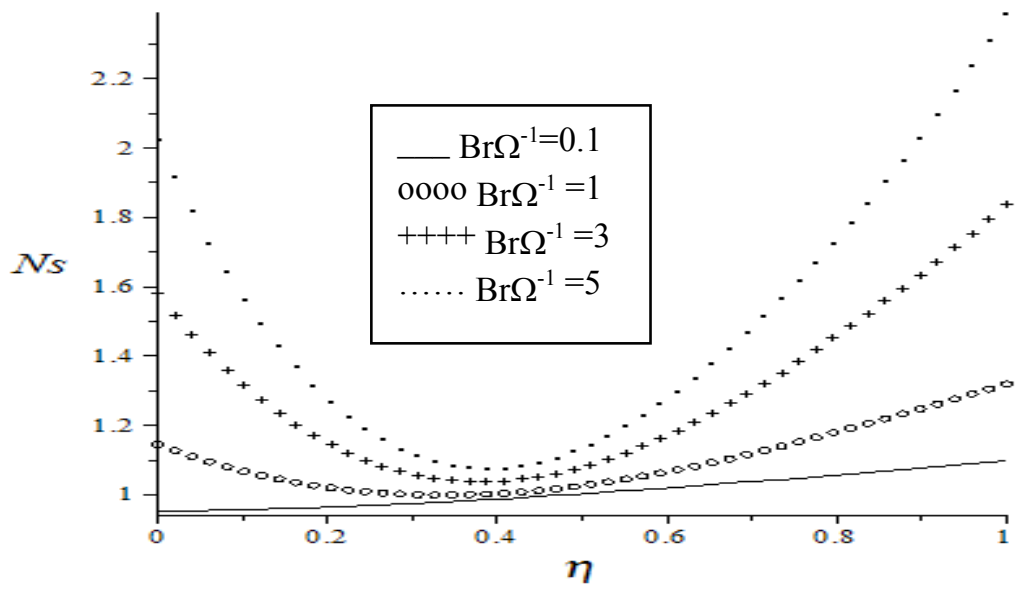

Figure 10: Entropy generation rate, $\beta_{1}=\beta_{2}=0.1, G=1, \operatorname{Re}=0.1, \operatorname{Pr}=0.71$

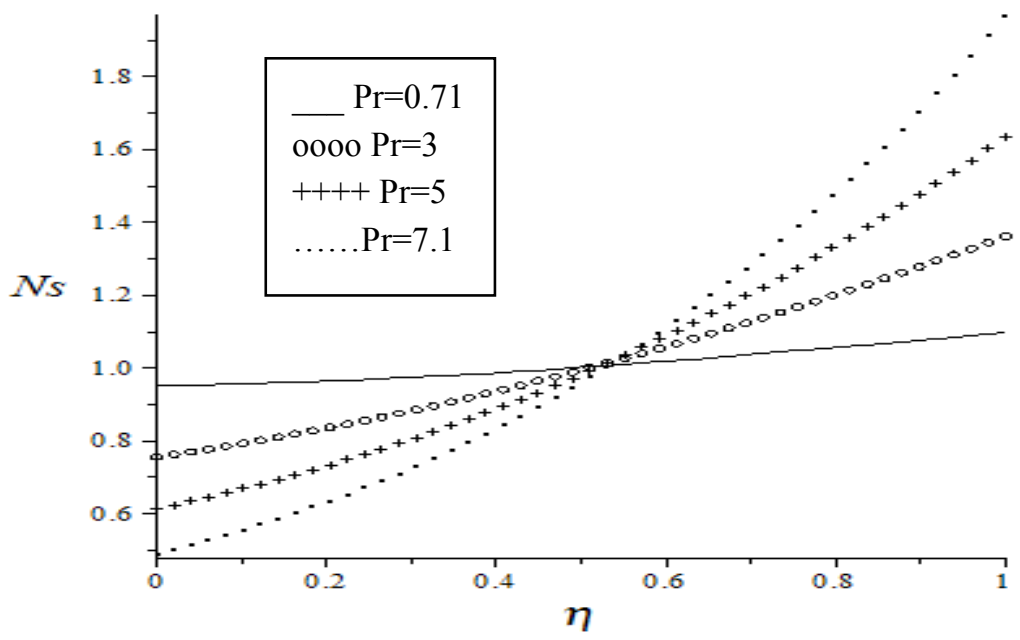

Figure 11: Entropy generation rate, $\operatorname{Br} \Omega^{-1}=\beta_{1}=\beta_{2}=0.1, G=1, \operatorname{Re}=0.1$ 


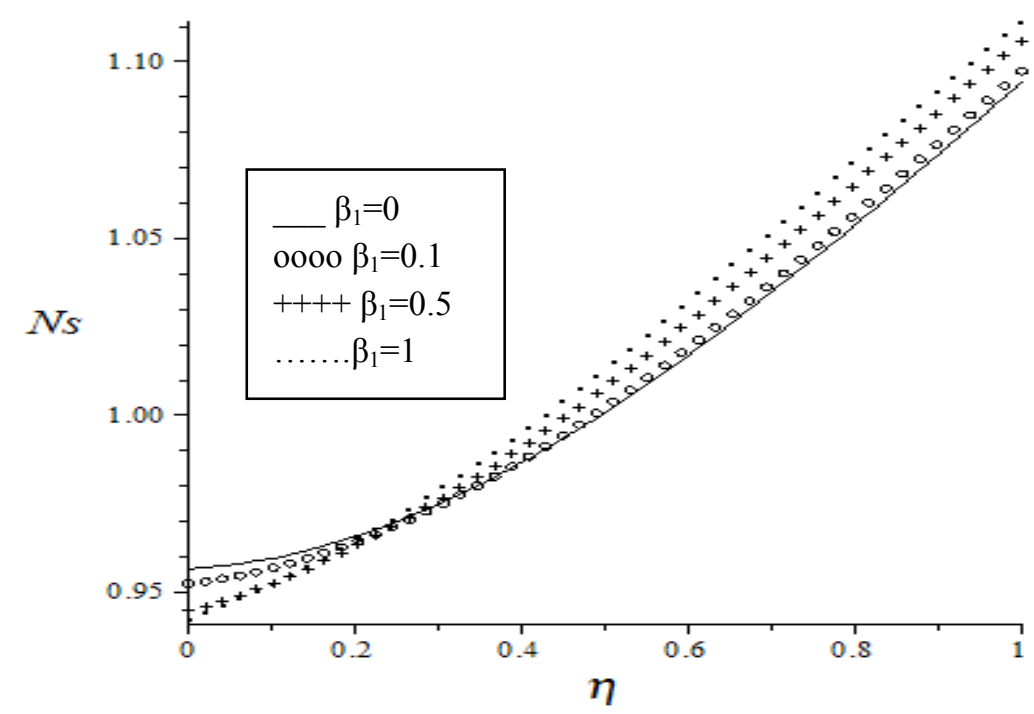

Figure 12: Entropy generation rate, $\operatorname{Br} \Omega^{-1}=\beta_{2}=0.1, G=1, \operatorname{Re}=0.1, \operatorname{Pr}=0.71$

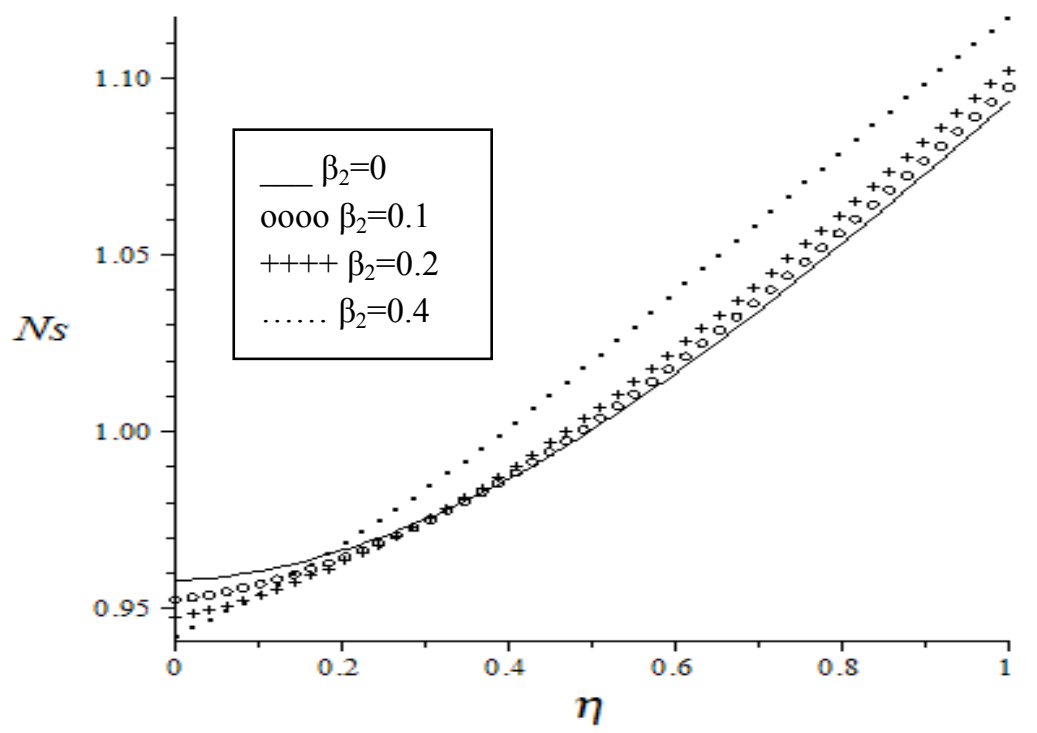

Figure 13: Entropy generation rate, $\operatorname{Br} \Omega^{-1}=\beta_{1}=0.1, G=1, \operatorname{Re}=0.1, \operatorname{Pr}=0.71$

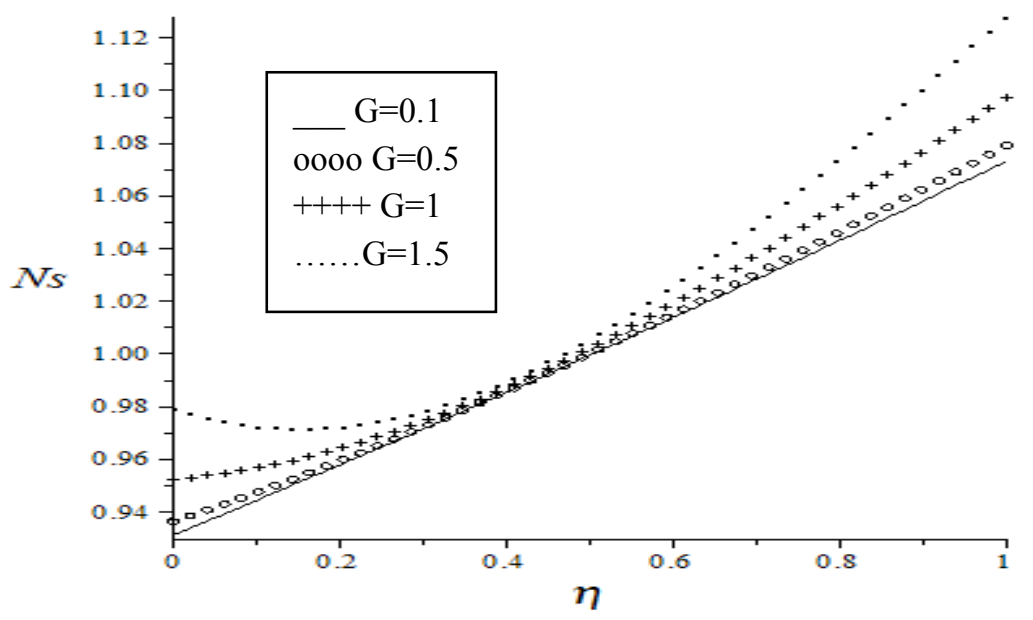

Figure 14: Entropy generation rate, $\operatorname{Br} \Omega^{-1}=\beta_{1}=\beta_{2}=0.1, \operatorname{Re}=0.1, \operatorname{Pr}=0.71$ 
Figs. 15-20 illustrate the effects of various thermophysical parameters on the Bejan number. Generally we noticed that heat transfer irreversibility dominates the flow process within the channel centerline region with Bejan number very close to 1 , while the little influence of fluid friction irreversibility can be observed at the channel walls. This perfectly agrees with the observation of Ajibade et al. (2011). In Fig.15, we observed that increase in Reynolds number; decreases the Bejan number on injection wall and increases the Bejan number on suction wall. This shows clearly that the irreversibility due to fluid friction tends to dominate the flow system at injection wall while heat transfer irreversibility dominates at the suction wall. Fig.16 depicts the effects of increasing group parameter; the Bejan number at both walls decrease leading to increasing influence of fluid friction irreversibility. Figs.17-18 demonstrate the effects of asymmetric slip coefficients $\beta_{1}$ and $\beta_{2}$. The Bejan number at injection wall increases while it decreases at the suction wall leading to the increasing influence of heat transfer irreversibility at the lower wall and fluid friction irreversibility at the upper wall. Increase in Prandtl number leads to decrease in Bejan number at injection wall and an increase in Bejan number at the suction wall (see Fig. 19). Consequently, the fluid friction irreversibility tends to dominate the flow system at injection wall while heat transfer irreversibility dominates at the suction wall. Fig. 20 shows that an increase in axial pressure gradient causes the influence of fluid friction irreversibility at both walls to increase greatly.

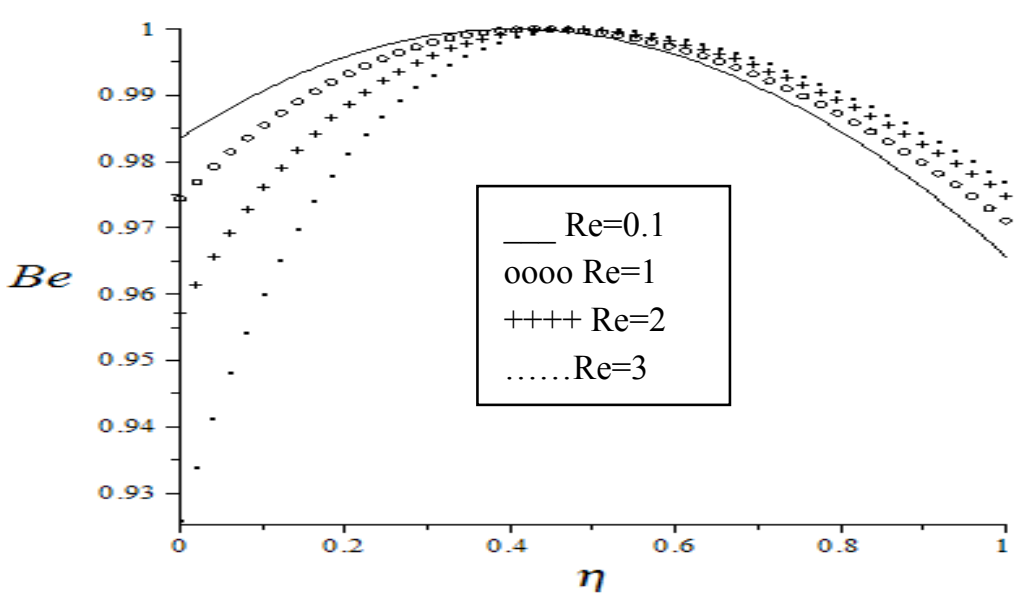

Figure15: Bejan Number, $\mathrm{Br} \Omega^{-1}=\beta_{1}=\beta_{2}=0.1, \mathrm{G}=1, \mathrm{Pr}=0.71$

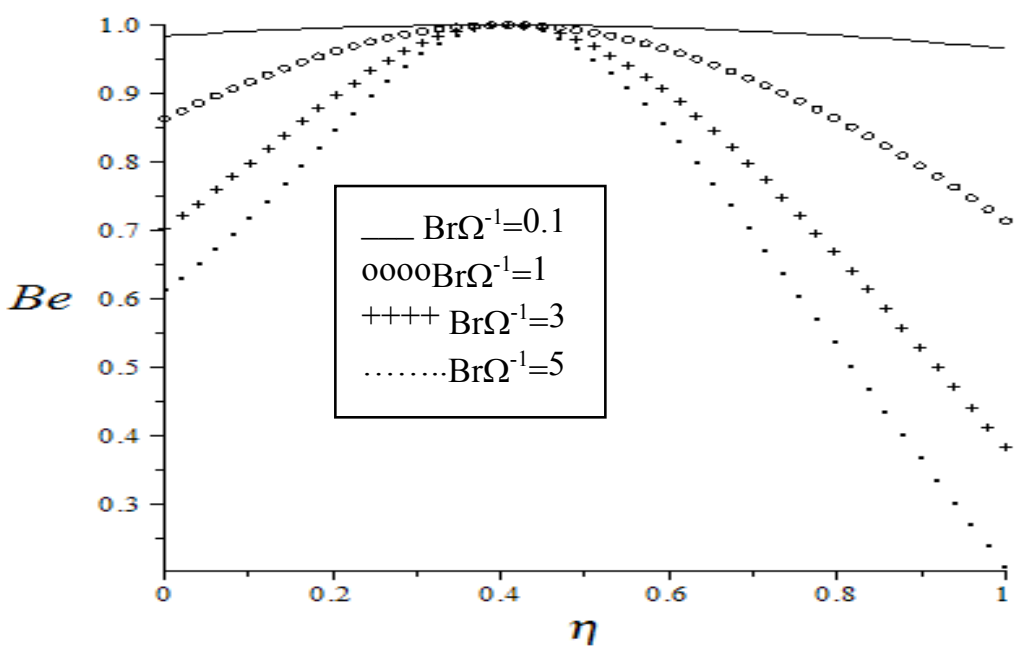

Figure16: $B$ jan number, $\beta_{1}=\beta_{2}=0.1, G=1, \operatorname{Re}=0.1, \operatorname{Pr}=0.71$ 


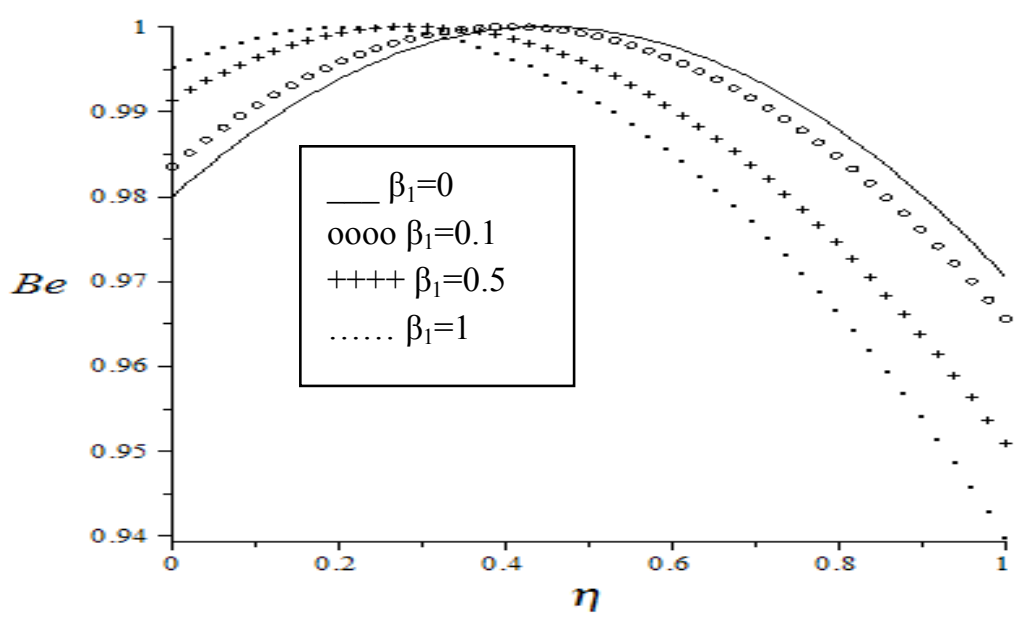

Figure 17: Bejan number, $\mathrm{Br} \Omega^{-1}=\beta_{2}=0.1, \mathrm{G}=1, \mathrm{Re}=0.1, \operatorname{Pr}=0.71$

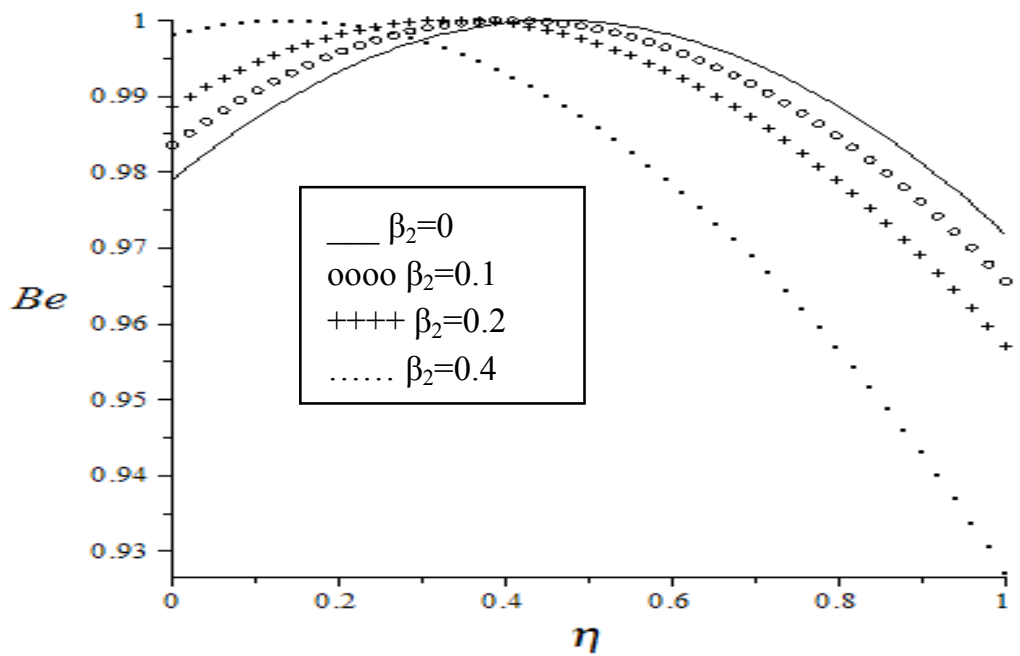

Figure 18: Bejan number, $\operatorname{Br} \Omega^{-1}=\beta_{1}=0.1, \mathrm{G}=1, \operatorname{Re}=0.1, \operatorname{Pr}=0.71$

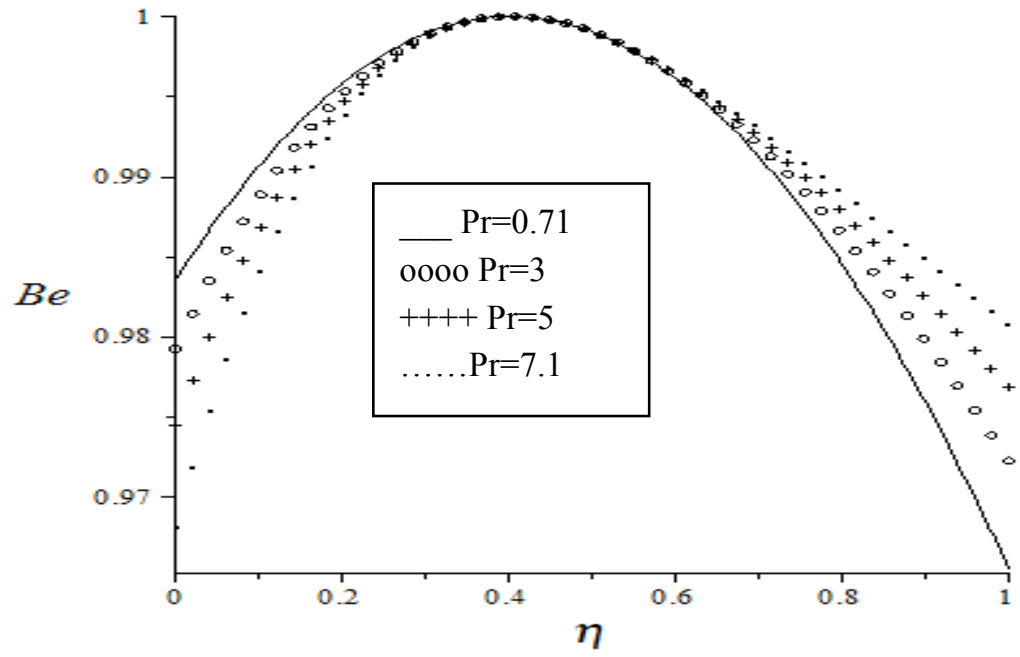

Figure 19: Bejan number, $\mathrm{Br} \Omega^{-1}=\beta_{1}=\beta_{2}=0.1, \mathrm{G}=1, \mathrm{Re}=0.1$ 


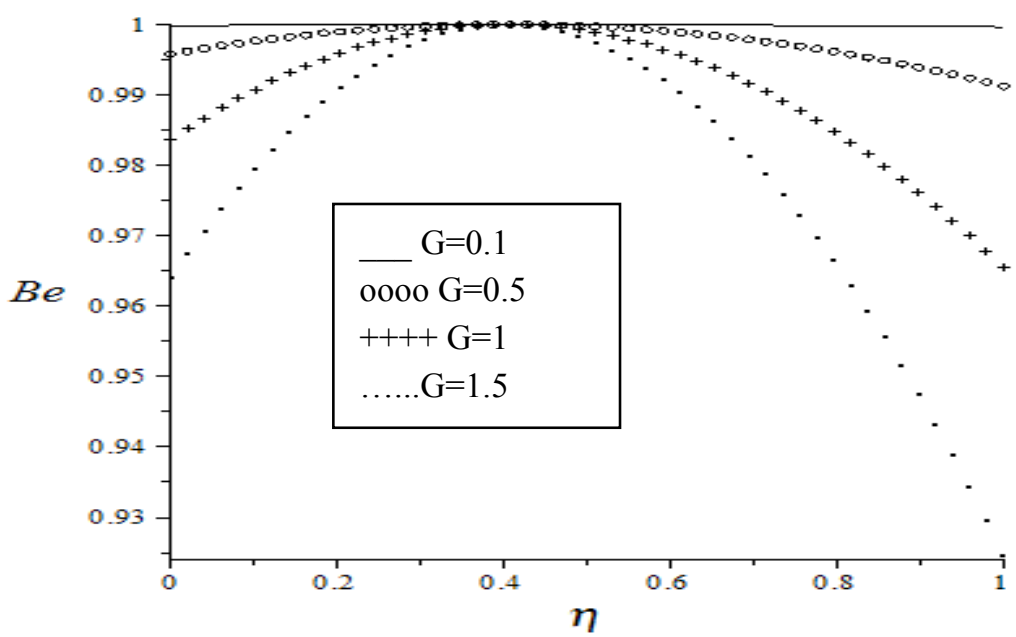

Figure 20: Bejan number, $\operatorname{Br} \Omega^{-1}=\beta_{1}=\beta_{2}=0.1, \operatorname{Re}=0.1, \operatorname{Pr}=0.71$

\section{Conclusions}

We analyzed in this paper, the combined effects of suction/injection and asymmetric Navier slips on the entropy generation rate in a steady flow of an incompressible fluid through a porous channel with non uniform walls temperature, putting into consideration that these will be of help in upgrading the system in terms of energy loss. Our results revealed among others the presence of flow reversal at the channel's upper wall due to suction. The heat transfer irreversibility dominates the flow process within the channel centerline region, while the influence of fluid friction irreversibility can be observed at the channel walls. However, as the values of asymmetric slip parameters increase, the dominance effects of heat transfer irreversibility at the lower wall and fluid friction irreversibility at the upper wall increase. Entropy generation minimization has become most important area of research for scientists and engineers in this crucial time. Moreover, with appropriate combination of thermophysical parameter values, entropy minimization can be achieved in a flow process.

\section{References}

(1) Ajibade,A.O.,B.K, Jha and A.Omame. "Entropy Generation under the effect of suction/injection". Applied Mathematical Modelling. 35, 4630-4046. (2011)

(2) Bejan, A. "Second-Law analysis in heat transfer and thermal design". Adv. Heat Transfer Vol.15, pp1-58.(1982)

(3) Bejan, A. "Entropy Genaration Minimization", CRC, Boca Raton, NY.(1996)

Berman A. S. "Laminar flow in channels with porous walls". J. Appl. Phys. 24, 1232-1235. (1953)

(4) Berh,M. "On the Application of Slip Boundary Condition on Curve Boundaries".Int.J for Numerical Methods in fluid. 45, 43-51.(2004)

(5) Buckingham,R.,M. Shearer and A. Bertozzi. "Thin Film Travelling Waves and THE Navier Slip Condition”. SIAM J.APPL MATHS vol 63, No2 pp722-744.(2003)

(6) Brady, J. F., "Flow development in a porous channel and tube" Phys. Fluids, 27, 1061-1067, (1984).

(7) Chauhan,D.S. and V.Kumar,V. "Effect of Slip condition on forced convection and entropy generation in a circular channel occupied by highly porous medium: Darcy extended Brinkmann-Forchheimer model”. Turkish J.Eng.Env. Sci 33, 91-104. (2009) 
(8) Erbay,L.B.,M.S Ercan ,B.Sulus and Yalcin,M.M. "Entropy Generation during Fluid Flow between two Parallel plates with moving Bottom plate". Entropy, 5, 506-516. (2003)

(9) Gupta,M. "Effect of wall Slip on the Flow in a flat Die for Sheet Extrusion". ANTEC pp1191-1196. (2011)

(10) Haddad,O., M.Abuzaid., and M.Al-Nimir. "Entropy generation due to Laminar Incompressible forced convection flow through parallel-plates micro channel". Entropy, 6(5), 413-426.(2004)

(11) Makinde,O.D., and A.Aziz. " Second law analysis for a variable viscosity plane Poiseuille flow with asymmetric convective cooling”. Computers and Mathematics with Applications, Vol 60, pp3012-3019. (2010)

(12) Makinde, O.D. "Thermodynamic second law analysis for a gravity driven variable viscosity liquid film along an inclined heated plate with convective cooling”. Journal of Mechanical Science and Technology, Vol. 24 (4), pp899-908 (2010)

(13) Makinde, O.D. "Extending the utility of perturbation series in problems of laminar flow in a porous pipe and diverging channel".J.Austral.Math.Soc.Ser B 40,1-11 (1998).

(14) Makinde, O. D. "On MHD mixed convection with Soret and Dufour effects past a vertical plate embedded in a porous medium". Latin American Applied Research, 41, 63-68, (2011).

(15) Nachtshein.P.R. and P.Swigert. "Satisfaction of the Asymptotic Boundary Conditions in Numerical Solution of the System of Nonlinear Equation of Boundary Layer Type.”NASA TND-3004, 1965.

(16) Navier. CLMH. "M'emoire sur les lois du mouvement des fluides. Mem." Acad. R. Sci. Paris., 6:389-416, 1823.

(17) Ozalp,A.A "Combined effects of pipe Diameters Reynlods number and wall heat flux and on flow, heat Transfer and Second law characteristics of laminar-Transitional micro-pipe flows". Entropy, 12, 445-472. (2010)

(18) Raoufpanah,A. "Effects of Slip Condition on the characteristics of flow in ice melting process". Int.J of Engineering, Vol.18, No.3, 1-9. (2005)

(19) Sahraoui,M. and M. Kaviany "Slip and no-slip temperature boundary conditions at the interface of porous, plane media: Convection". Int.J Heat Transfer Vol.37, No6,pp 1029-1044.(1994)

(20) Sahin, A.Z., and R. Ben-Mansom. "Entropy generation in laminar fluid flow through a circular pipe".Entropy, 5, 404-416.(2003)

(21) Sahin, A.Z. "Entropy generation in turbulent liquid flow through a smooth duct subjected to constant wall temperature".Int.J.Heat and Mass Transfer, 43, 11469-1478.(2000)

(22) Terrill $R$, M. "Laminar flow in a uniformly porous channel”. Aeronaut. $Q$. 15, 299-310. (1964)

(23) Terrill $R$, M. "Laminar flow in a uniformly porous channel with large injection". Aeronaut. Q. (1965)

(24) Tripathi, D.,P.K.Gupta and S. Das, "Influence of slip condition on peristaltic transport of a viscoelastic fluid with fractional Burgens model”. Thermal Science, Vol.15, No.2, 501-515. (2011)

(25) Vazquez, F., M.A.Olivare-Robles and S. Medina "Size effects on the entropy production in oscillation flow between parallel plates". Entropy, 13, 542-553. (2011) 